\title{
Editorial: Machine Learning and Intelligent Communications
}

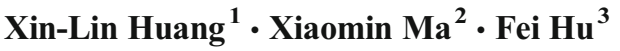

Published online: 28 October 2017

(C) Springer Science+Business Media, LLC 2017

\section{Editorial:}

Along with the fast developing of mobile communications technologies, the amount of high quality wireless services is required and increasing exponentially. According to the prediction of Cisco VNI Mobile Forecast 2016, global mobile data traffic will increase nearly eightfold between 2015 and 2020, and mobile network connection speeds will increase more than threefold by 2020 . Hence, there is still a big gap between the future requirements and current communications technologies, even using 4G/5G. How to integrate the limited wireless resources with some intelligent algorithms/schemes and boost potential benefits are the interests of the conference. As an emerging discipline, machine learning is a subfield of computer science that evolved from the study of pattern recognition and computational learning theory in artificial intelligence, and explores the study and construction of algorithms that can learn from and make predictions on complicated scenarios. In communication systems, the previous/current radio situations and communication paradigms should be well considered to obtain a high quality of service (QoS), such as available spectrum, limited energy, antenna configurations, and heterogeneous properties. Machine learning algorithms facilitate complicated scenarios analysis and prediction, and thus to make an optimal actions in OSI seven layers. We hope

Xin-Lin Huang

xlhuang@tongji.edu.cn

1 Department of Information and Communication Engineering, Tongji University, Shanghai, China

2 Department of Computer Science, Oral Roberts University, Tulsa, OK, USA

3 Department of Electrical and Computer Engineering, University of Alabama, Tuscaloosa, AL, USA

the integrating of machine learning algorithms into communication systems will improve the QoS and make the systems smart, intelligent, and efficient. We invite high quality original research papers describing recent and expected challenges or discoveries along with potential intelligent solutions for future mobile communications and networks. We welcome both theoretical and experimental papers. We expect the papers of the special issue to serve as valuable references for a large audience from both academia and industry. Both original, unpublished contributions and survey/tutorial types of articles are encouraged.

This special issue features six selected papers with high quality. The first article titled "Community Detection Based on Regularized Semi-Nonnegative Matrix Tri-Factorization in Signed Networks" from Zhen Li, Jian Chen, Ying Fu, Guyu $\mathrm{Hu}$, Zhisong Pan and Liangliang Zhang, proposed a framework based on regularized semi-nonnegative matrix trifactorization which mapped the signed network from highdimensional space to low-dimensional space. Then, the communities of the signed network could be derived. In addition, to improve the detection accuracy, they introduced a graph regularization to distribute the pair of nodes which were connected with negative links into different communities. Experiments on both synthetic data and real-world data all demonstrated that the proposed ReS-NMF had better performance and regularization parameter sensitivity compared with other algorithms.

The second article titled "Physical-Layer Network Coding Scheme over Asymmetric Rayleigh Fading Two-Way Relay Channels" from Bo Li, Xuesong Ding, Hongjuan Yang, Gongliang Liu and Xiyuan Peng, studied a new PNC scheme named bi-quadrature physical-layer network coding (BQPNC) for Rayleigh flat fading TWRC. In this paper, the authors gave the BER analysis of BQ-PNC and evaluated its performance by simulation. Theoretical and simulation results 
showed that the proposed scheme could significantly enhanced the BER performance, either in symmetric or asymmetric cases. Especially for uplink asymmetric TWRC, BQ$\mathrm{PNC}$ could provide more than $4 \mathrm{~dB}$ gain compared with $\mathrm{PNC}$ scheme.

The third article titled "Dynamic Provider Selection \& Power Resource Management in Competitive Wireless Communication Markets" from Panagiotis Vamvakas, Eirini Eleni Tsiropoulou and Symeon Papavassiliou, considered the combined problem of Wireless Internet Service Provider (WISP) selection by the mobile customers and corresponding power allocation. Each WISP was characterized by a price and service-based reputation, formed based on its adopted pricing policy and its success to satisfy customers' Quality of Service (QoS) prerequisites. The mobile customers acted as learning automata, who sensed their environment and took the best decision regarding the WISP to be served from. The optimal power allocation was concluded from the maximization problem of each user's utility function, which was treated as a non-cooperative game among users. A two-stage iterative algorithm was proposed in order to realize the machine learning provider selection and the distributed resource allocation.

The fourth article titled "Improved KMV-Cast with BM3D Denoising" from Xin-Lin Huang, Xiaowei Tang, Xiaoning Huan, Ping Wang and Jun Wu, proposed to use the wellknown block-matching and three dimensional filtering (BM3D) algorithm to remove noise for KMV-Cast. BM3D was realized by block-matching, collaborative filtering, aggregation, and Wiener filtering. In this paper, BM3D was used to remove noise after videos/images reconstruction using KMVCast scheme at receiver. The block-matching was used to collect similar pixel groups to reveal the property of the patch, while the collaborative filtering preserved the unique feature of each block through hard-thresholding. The aggregation process performed averaging to get the estimation results. The simulation results showed that KMV-Cast with BM3D denoising performed the best compared with other two algorithms including SoftCast and KMV-Cast, especially in case of low channel quality and all image blocks are needed to be transmitted.

The fifth article titled "Robust Localization with Crowd Sensors: A Data Cleansing Approach" from Changju Kan, Guoru Ding, Qihui Wu and Tao Zhang, investigated the source localization problem with crowd of anchor nodes under the circumstances that abnormal data could be sporadically and randomly produced for the reason of either accidental equipment failures or random malicious behaviors. The authors formulated a generalized modeling of abnormal data in localization problem, which considered the impacts of both unexpected equipment failures and malicious data falsifications. The corresponding Cramer-Rao lower bound (CRLB) of the specific localization problem was given. For the localization enhancement, the authors proposed a data cleansingbased robust localization algorithm which exploited the low occupancy of channel band by sources and the sparsity of abnormal data. The root mean squared error (RMSE) was derived to assess the performance of the proposed robust localization algorithm. Simulation results demonstrated that the proposed data cleansing-based robust localization algorithm could effectively eliminate the impairment caused by abnormal data and improve the localization performance.

The last article titled "Scheduling for Data Transmission in Multi-Hop IEEE 802.15.4e TSCH Networks" from Meng Mei, Yujun Zhu, Dadong Zhao and Fan Chen, studied the problem of data transmission scheduling for TSCH network which used the IEEE802.15.4e TSCH as the MAC protocol. The authors adopted matching rule to reduce conflicts in the TSH network and matching scheduling algorithm (MSA) to address the problem of communication resources allocation in tree topology. The scheduling algorithm MSA could avoid conflict and reduce transmission delay and energy consumption. The simulation results were shown to prove the effectiveness of the proposed method.

Acknowledgements The guest editors would like to thank all reviewers for their efforts in reviewing manuscripts submitted to this special issue. We also thank the Edit-in-Chief, Dr. Imrich Chlamtac for his supportive guidance during the entire process. The special issue is supported by the National Natural Science Foundation of China under Grant No. U1733114 and No. 61631017.

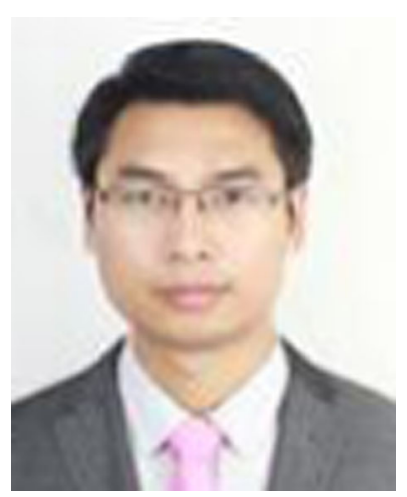

Xin-Lin Huang ( $S^{\prime} 09-M^{\prime} 12$ SM'16, IEEE) received the M.E. and $\mathrm{Ph} . \mathrm{D}$. degrees in information and communication engineering from Harbin Institute of Technology (HIT), Harbin, P. R. China, in 2008, and 2011, respectively. Right now, he is an Associate Professor with the Department of Information and Communication Engineering, Tongji University, Shanghai, P. R. China. His research focuses on Cognitive Radio Networks, Multimedia Transmission, Machine Learning, OFDM Technology, Massive MIMO Feedback. He has published over 60 research papers, 4 patents, and 3 book chapters in these fields. Dr. Huang was a recipient of Scholarship Award for Excellent Doctoral Student granted by Ministry of Education of China in 2010, Best PhD Dissertation Award from HIT in 2013, Shanghai High-level Overseas Talent Program in 2013, and Shanghai "Chenguang" Scholar Program in 2014. From Aug. 2010 to Sept. 2011, he was supported by China Scholarship Council to do research in the Department of Electrical and Computer Engineering, University of Alabama (USA), as a visiting scholar. He was 
invited to serve as Session Chair for the IEEE ICC2014. He served as a Guest Editor for IEEE Wireless Communications and Chief Guest Editor for International Journal of MONET. He also serves as IG Co-chair for IEEE ComSoc-MMTC. He is a Senior Member of the IEEE.

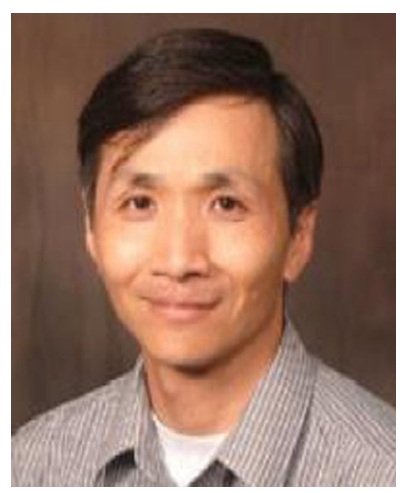

Xiaomin Ma (M'03-SM'08, IEEE) received B.E. and M.E. degrees in electrical engineering in 1984 and 1989, respectively. He got the Ph.D. degree in Information engineering at the Beijing University of Posts \& Telecommunications, China, in 1999. From 2000 to 2002, he was a post-doctoral fellow in the Department of Electrical and Computer Engineering, Duke University, USA. He had been teaching in the field of Electrical and Computer Engineering as an assistant professor and associate professor at the Petroleum University of China for about eight years. Then, he worked in a telecommunication company (Huawei in Beijing) for a short time. Currently, he is a professor in the Department of Engineering at Oral Roberts University in U.S. He has published over 100 papers in peer-reviewed journals and conferences. $\mathrm{He}$ also holds a US patent. He is the recipient of Best Paper Award in IEEE International Conference on Network Infrastructure and Digital Content. He is in Editorial Board of International Journal of Vehicular Technology, Hindawi Publish House. Also, he is a guest editor of Special
Issue on "Reliable and secure VANETs" in IEEE Transactions on Dependable and Secure Computing and a guest editor of Special Issue on " Emerging Technologies in Wireless Communications" in ACM/ Springer Mobile Networks \& Applications (MONET). His research interests include stochastic modeling and analysis of computer and communication systems, physical layer and MAC layer of vehicular ad hoc wireless networks, computational intelligence and its applications to coding, signal processing, and control, and Quality of service (QoS) and call admission control protocols in wireless networks. He is (or was) PI, Co-PI or project leader in several projects sponsored by NSF, NSF EPSCoR, Motorola, Chinese NSF, AFOSR, and ARO, etc. Currently, he is a senior member of the IEEE.

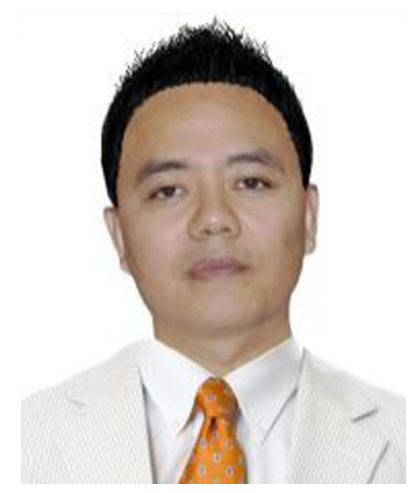

Fei Hu (M'12, IEEE) received the Ph.D. degrees in signal processing from Tongji University, Shanghai, China, in 1999, and in electrical and computer engineering from Clarkson University, New York, NY, USA, in 2002. $\mathrm{He}$ is currently a professor with the Department of Electrical and Computer Engineering at the University of Alabama (main campus), Tuscaloosa, AL, USA. His research expertise is cognitive radio networks and security. $\mathrm{He}$ has published over 170 journal/ conference papers and book chapters. His research has been supported by the U.S. the NSF, Cisco, Sprint, and other sources. 